\title{
Analysis of Nanodipoles in Optical Nanocircuits Fed by Gaussian Beam
}

\author{
Karlo Costa, Victor Dmitriev, Janilson Souza, and Gustavo Silvano \\ Department of Electrical Engineering, Federal University of Para, Avenida Augusto Corrêa No. 01, 66075-900 Belém, PA, Brazil \\ Correspondence should be addressed to Karlo Costa; karlo@ufpa.br
}

Received 24 November 2013; Revised 11 April 2014; Accepted 12 April 2014; Published 17 June 2014

Academic Editor: Hon Tat Hui

Copyright ( 2014 Karlo Costa et al. This is an open access article distributed under the Creative Commons Attribution License, which permits unrestricted use, distribution, and reproduction in any medium, provided the original work is properly cited.

\begin{abstract}
We analyze impedance matching and excitation properties of a plasmonic optical nanocircuit composed by a receiving and an emitting dipole connected by a two-wire optical transmission line. The circuit is fed by a circular Gaussian beam focused on the receiving dipole. The numerical analysis is performed by linear method of moments with a given surface impedance of gold cylindrical conductors. With this model, we analyze the variation of standing-wave response along the circuit in function of some geometrical parameters. We present some conclusions concerning impedance matching between the transmission line and the emitting dipole and coupling between the receiving dipole and the incident Gaussian beam.
\end{abstract}

\section{Introduction}

Optical antennas are metal nanostructures used to transmit or receive optical fields [1-4]. This definition is similar to that of conventional antennas in RF-microwave regimes. However, optical antennas can also be used to enhance and confine optical fields in nanometer regions smaller than the operating wavelength, that is, beyond the diffraction limit of light. This is possible due to the plasmonic effect of metals in optical frequencies which produces strong electrons oscillations named plasmonic resonances $[5,6]$.

The concept of optical antennas was firstly used in design of efficient near-field optical probes. Classical papers about this application are [7], where a microwave version of optical probe based on nanoantenna was experimentally analyzed, and [8], where several configurations and new ideas of optical probes based on optical antennas were theoretically presented. More recent experimental and theoretical works on optical probes based on nanoantennas can be found in $[9,10]$. Other examples of applications are enhancement of single molecule fluorescence [11-14], ultrahigh-density optical data storage devices [15], chemical enhancement for surface-enhanced Raman scattering [16], nanobioimaging [17], enhancement in the directional excitation and emission of single emitters $[18,19]$, plasmonic laser antennas [20], and plasmonic photovoltaic cells $[21,22]$.
Potential applications of optical antennas and plasmonic waveguides are in nanophotonics. These elements can be used, for example, as fundamental components for the design of highly integrated photonic signal-processing systems, because they can manipulate optical fields in subwavelength nanoscales, beyond the diffraction limit [23]. In this case, plasmonic waveguides can be used to interconnect different devices in an optical nanocircuit, and optical antennas can act as terminal elements that transform far field radiation into guided waves and vice versa. Some examples of plasmonic waveguide are two-wire optical transmission line (OTL) [24] and array of nanoparticles, acting as lumped nanocircuit elements [25].

Optical nanocircuits based on two-wire OTL and nanoantennas have been investigated in [26, 27]. In [26], a theoretical analysis of an optical nanocircuit composed by a receiving and an emitting nanoantenna connected by a two-wire OTL is presented. In this paper, the authors performed the input impedance matching varying the length of the nanodipoles for a fixed frequency, radius, and gap of the OTL. In [27], analysis of an optical nanocircuit fed by an aperture probe is fulfilled. The circuit is composed by a receiving and an emitting nanoantenna connected by a two-wire OTL and the coupling between the aperture probe and the receiving dipole is modeled by an equivalent voltage source. 
In this paper, we present an impedance matching and excitation analysis of a plasmonic optical nanocircuit composed by a receiving and an emitting nanodipoles connected by a two-wire OTL. The feeding of this circuit is made by an aperture probe that illuminates the receiving dipole. This circuit is similar to that presented in [27], but in the present case we model the radiated field from the aperture probe by a focused Gaussian beam [28], with $\lambda=830 \mathrm{~nm}$, beam waist of $340 \mathrm{~nm}$, and electric field with polarization matched to the axis of the receiving dipole. The numerical analysis of this circuit is made by a simple and efficient computational method based on method of moments (MoM) [27]. We study the variation of the induced current, voltage reflection coefficient and near-field distribution for different length and radius of the receiving and emitting dipole, and different radius and gap of the OTL. We present some conclusions about the conditions where one can obtain a better impedance matching between the emitting dipole and the OTL. We also discuss the conditions which allow one to obtain an efficient electromagnetic coupling between the Gaussian beam and the receiving dipole. The next sections present the theoretical development, impedance matching analysis, excitation analysis, and conclusions.

\section{Theoretical Development}

This section presents the geometry of the problem, the linear method of moments (MoM) model of the circuit, the Gaussian beam used to feed the circuit, and a numerical example of simulation.

2.1. Description of the Problem. The analyzed optical circuit is shown in Figure 1 where an aperture probe is electromagnetically coupled to a nanocircuit. The nanocircuit is composed of a receiving dipole, two-wire optical transmission line, and an emitting dipole. The material of the circuit is gold and the medium is vacuum with no substrate. We model the coupling between the aperture probe and the receiving antenna by an equivalent focused Gaussian beam radiated from the aperture. The polarization of this field is parallel to the axis of the receiving dipole (axis $x$ ) to maximize the electromagnetic coupling and efficiency.

We calculate the impedance matching between the OTL and the emitting dipole and optimize the electromagnetic coupling between the receiving dipole and the Gaussian beam. We solve numerically the scattered problem by the method of moments and calculate the induced current, voltage reflection coefficient, and near-field distribution.

2.2. Method of Moments Model. To analyze the equivalent nanocircuit of the system presented in Figure 1, we use the linear method of moments (MoM) approximation with sinusoidal basis functions and equivalent surface impedance [27]. Figure 2 shows the geometry of the original problem and the MoM equivalent model. In Figure 2(a), we depicted receiving dipole 1 (left), an OTL, and an emitting dipole 2 (right).
The dimensions of this circuit are $h_{1}, a_{h 1}$ and $h_{2}, a_{h 2}$ the arm length and radius of the nanodipoles 1 and 2, respectively, $L$ and $a_{L}$ the length and radius of the OTL wires, respectively, $d$ the distance between the axes of the OTL, and $D=d-2 a_{L}$ the gap distance between the surfaces of the OTL. The total length of nanodipoles 1 and 2 is $H_{1}=2 h_{1}+d$ and $H_{2}=2 h_{2}+d$, respectively.

In the scattering problem of Figure 1, the gold material of the cylindrical conductors of the circuit is represented by the Lorentz-Drude model for the complex permittivity $\varepsilon_{1}=\varepsilon_{0} \varepsilon_{r 1}$ [1], where

$$
\varepsilon_{r 1}=\varepsilon_{\infty}-\frac{\omega_{p 1}^{2}}{\omega^{2}-j \Gamma \omega}+\frac{\omega_{p 2}^{2}}{\omega_{0}^{2}-\omega^{2}+j \gamma \omega},
$$

$\varepsilon_{\infty}=8, \omega_{p 1}=13.8 \times 10^{15} \mathrm{~s}^{-1}, \Gamma=1.075 \times 10^{14} \mathrm{~s}^{-1}, \omega_{0}=$ $2 \pi c / \lambda_{0}, \lambda_{0}=450 \mathrm{~nm}, \omega_{p 2}=45 \times 10^{14} \mathrm{~s}^{-1}$, and $\gamma=9 \times 10^{14} \mathrm{~s}^{-1}$. The losses in metal are described by surface impedance $Z_{s}$. This surface impedance can be obtained approximately by considering cylindrical waveguide with the mode $\mathrm{TM}_{01}$ [29]:

$$
Z_{s}=\frac{T J_{0}(T a)}{2 \pi a j \omega \varepsilon_{1} J_{1}(T a)}
$$

where $T=k_{0} \sqrt{\varepsilon_{r 1}}$ and $k_{0}=\omega \sqrt{\mu_{0} \varepsilon_{0}}$. The boundary condition for the electric field satisfied at the surface's conductor is $\left(\bar{E}_{s}+\bar{E}_{i}\right) \cdot \bar{a}_{l}=Z_{s} I$, where $\bar{a}_{l}$ is a unitary vector tangential to the surface of the metal, $\bar{E}_{s}$ is the scattered electric field due to the induced linear current $I$ on the conductor, $\bar{E}_{i}$ is the incident electric field of the Gaussian beam source (Figure 1), and $I$ is the induced longitudinal current in a given point of the nanodipole.

The integral equation of the scattered field along the length $l$ of the nanodipole is given by [27]

$$
\bar{E}_{s}(\bar{r})=\frac{1}{j \omega \varepsilon_{0}}\left[k_{0}^{2} \int_{l} \bar{I} g(R) d l^{\prime}+\int_{l} \frac{d I}{d l^{\prime}} \nabla g(R) d l^{\prime}\right],
$$

where $g(R)=e^{-j k_{0} R} / 4 \pi R$ is the free space Green's function and $R=\left|\bar{r}-\bar{r}^{\prime}\right|$ is the distance between source and observation points. The numerical solution of the problem formulated by the boundary condition and (1)-(3) is performed by linear MoM as follows. Firstly, we discretize the linear circuit as shown in Figure 2(b), where $N_{L}, N_{h 1}$, and $N_{h 2}$ are the number of straight segments in $L, h_{1}$, and $h_{2}$, respectively. In this case, we have $N_{L}=7, N_{h 1}=N_{h 2}=3$. The discretization is uniform in $L, h_{1}$, and $h_{2}$, but the discretization length can be different; that is, $\Delta L_{L}=L / N_{L}, \Delta h_{1}=h_{1} / N_{h 1}$, and $\Delta h_{2}=h_{2} / N_{h 2}$. With this discretization, the total number of straight segments of the nanocircuit is $N_{t}=2 N_{h 2}+2 N_{L}+2 N_{h 1}$. For stability of the method, we use the convergence conditions $\Delta h_{2}>2 a_{h 2}$, $\Delta h_{1}>2 a_{h 1}$, and $\Delta L_{L}>2 a_{L}$. Then the current in each segment is approximated by sinusoidal basis functions [27]. The expansion constants $I_{n}$ are shown in Figure 2(b) where each constant defines one triangular sinusoidal current. To determine these constants, we use $N=N_{t}-2$ rectangular pulse test functions with unitary amplitude and perform the 


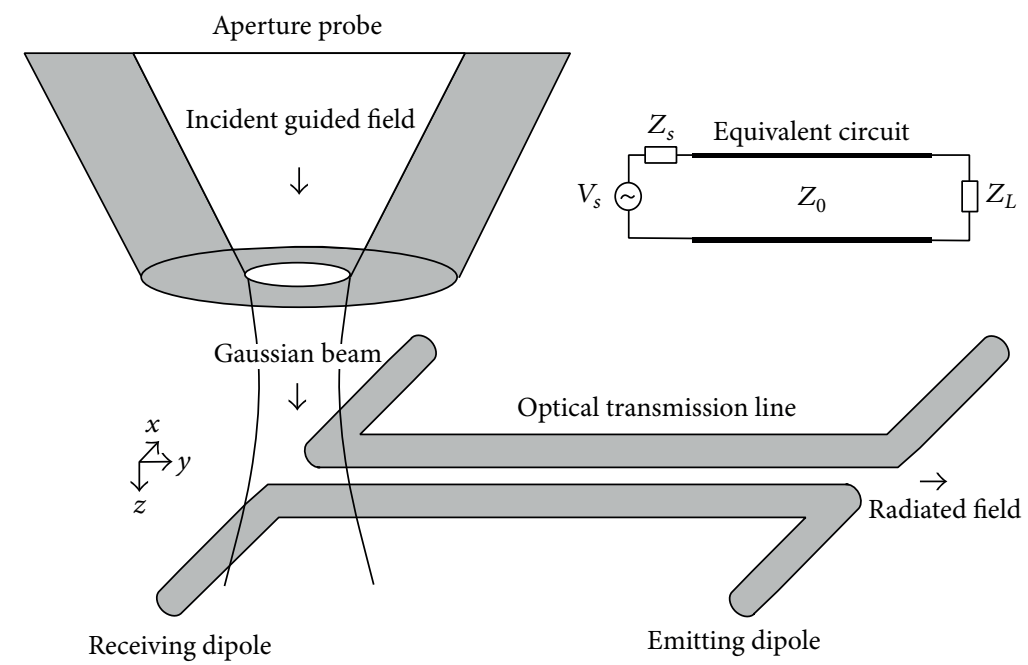

FIGURE 1: Aperture probe coupled to optical nanocircuit. Nanocircuit is formed by receiving dipole, optical transmission line, and emitting dipole.

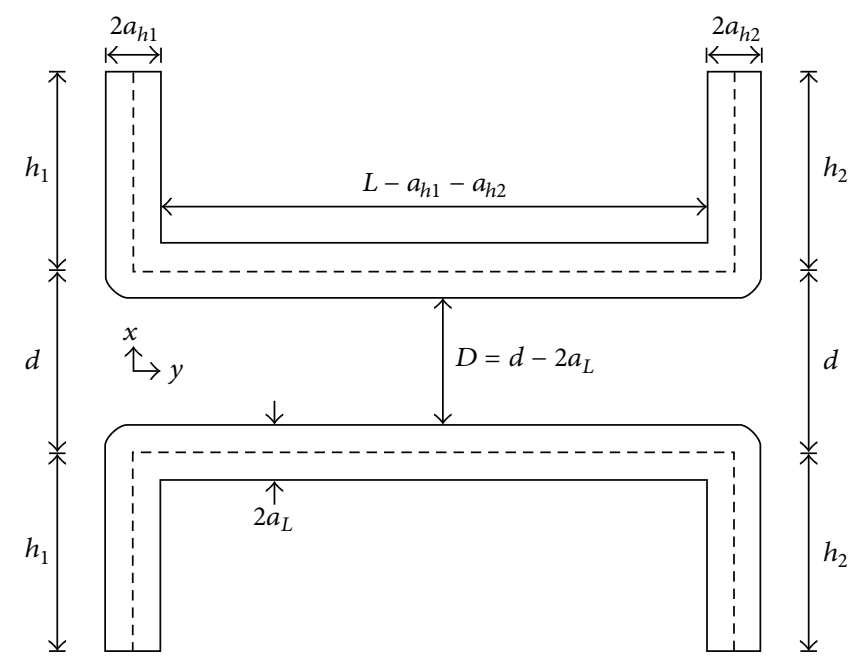

(a)

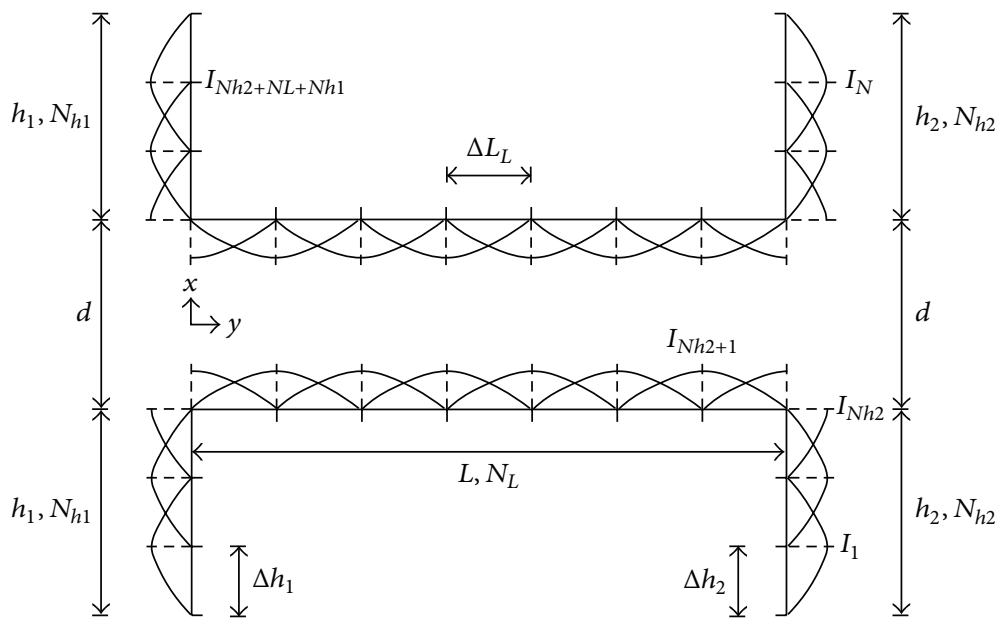

(b)

FIGURE 2: Cylindrical plasmonic nanocircuit composed of receiving (left) and emitting (right) nanodipoles and optical transmission line (middle). (a) Geometry of original problem. (b) Discretization used in MoM model. 

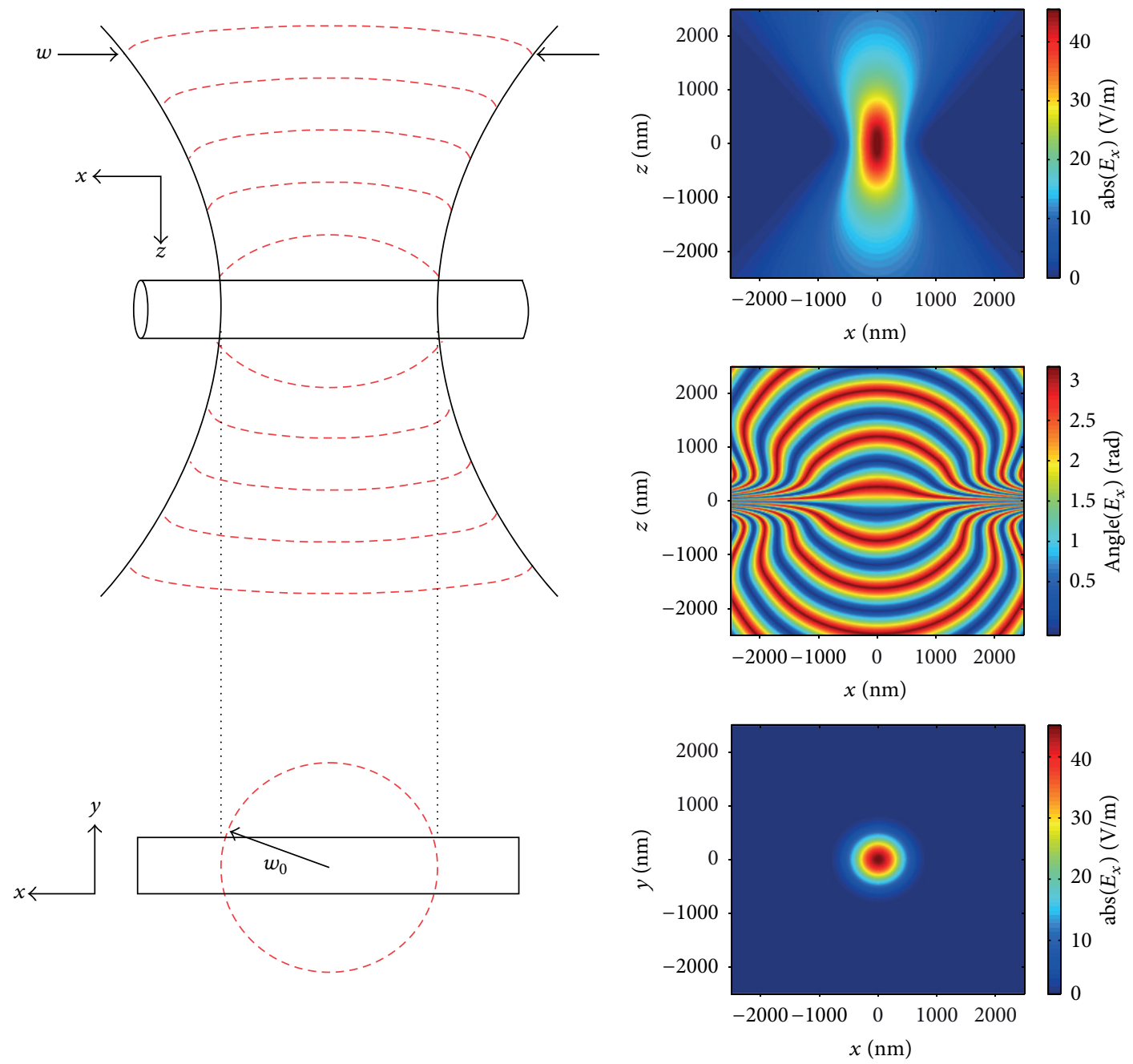

FIGURE 3: Field distribution of Gaussian beam with $P=10^{-12} \mathrm{~W}, \lambda=830 \mathrm{~nm}$, and beam waist $w_{0}=340 \mathrm{~nm}$ at planes $x z$ and $x y$.

conventional testing procedure. The following linear system of equations is obtained:

$$
V_{m}=Z_{s} I_{m} \Delta_{m}-\sum_{n=1}^{N} Z_{m n} I_{n}, \quad m=1,2,3, \ldots, N
$$

where $Z_{m n}$ is the mutual impedance between sinusoidal current elements $m$ and $n, \Delta_{m}=\left[\Delta L_{m}+\Delta L_{m+1}\right] / 2$. The solution of (4) produces the current along the nanocircuit. With these results, it is possible to calculate the near and far field distributions and other parameters.

2.3. Gaussian Beam Source. The electromagnetic wave radiated from the aperture probe of Figure 1 illuminates the receiving dipole. It can be approximated by a Gaussian beam. This kind of wave is obtained by solving the scalar Helmholtz wave equation with the paraxial approximation [28]. The magnetic vector potential of a Gaussian beam polarized in the axis $x$ and traveling in the $+z$ direction is given by

$$
\bar{A}=u_{0} \sqrt{\frac{2}{\pi}} \frac{1}{w} \exp \left(\frac{-\rho^{2}}{w^{2}}\right) \exp \left[-j\left(\frac{k \rho^{2}}{2 R}-\phi\right)\right] e^{-j k z} \bar{a}_{x}
$$

where $u_{0}=\left(2 P \mu_{0} / k \omega\right)^{1 / 2}, P$ is the power of the beam, $w$ is the beam radius (Figure 3 ), $R$ is the curvature radius of the phase front, and $\phi$ is the phase of the beam. The three principal parameters that define the beam are the power $P$, the radius of the beam waist $w_{0}$ (Figure 3 ), and the operating wavelength $\lambda$. With the vector potential (5), the $x$ component of electric field $E_{x}$ of the Gaussian beam can be obtained from the Maxwell equations [28].

The beam of Figure 1 is focused on the receiving dipole 1 with polarization along the dipole axis (axis $x$ ), the direction of propagation is $+z$, the beam axis is along the axis $z$, and the minimum waist $\left(w_{0}\right)$ is localized at $z=0$, which is the plane of the nanocircuit. In all the analysis presented in this work, we consider a fixed Gaussian beam with power $P=10^{-12} \mathrm{~W}$, wavelength $\lambda=830 \mathrm{~nm}$, and beam waist $w_{0}=$ 


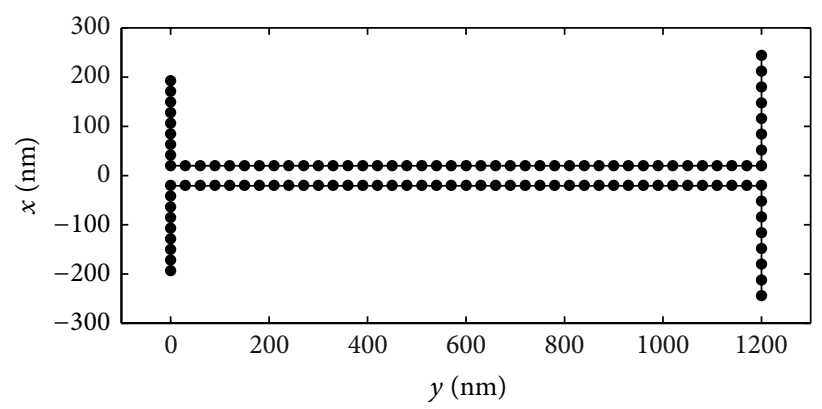

(a)

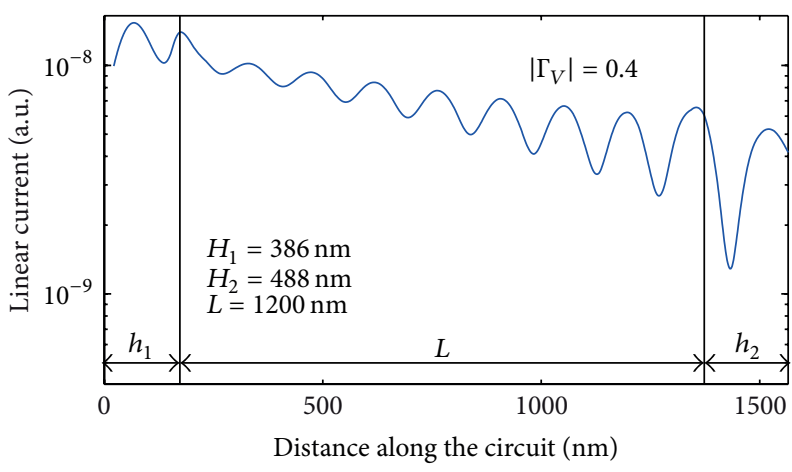

(b)

FIGURE 4: (a) Geometry and discretization of nanocircuit with $H_{1}=386 \mathrm{~nm}, H_{2}=488 \mathrm{~nm}, L=1200 \mathrm{~nm}, a_{h 1}=10 \mathrm{~nm}, a_{L}=a_{h 2}=15 \mathrm{~nm}$, $D=10 \mathrm{~nm}, N_{h 1}=8, N_{h 2}=7, N_{L}=40, \Delta L_{L}=30 \mathrm{~nm}, \Delta h_{1}=21.6 \mathrm{~nm}, \Delta h_{2}=32 \mathrm{~nm}$, and $N=108$. (b) Amplitude of linear current distribution along nanocircuit. The voltage reflection coefficient of this circuit is $\left|\Gamma_{V}\right|=0.4$.

$340 \mathrm{~nm}$. We use these values for comparisons with the results of paper [26], but the analysis presented here can be applied for beams with other parameters. The field distribution of this beam is shown in Figure 3, where the electric field amplitude $\operatorname{abs}\left(E_{x}\right)$ is presented at the planes $x z$ and $x y$ and the phase distribution angle of $E_{x}$ is presented at the plane $x z$. The phase at plane $x y$ is constant.

2.4. Numerical Example. Based on the theory presented above, we developed a MoM code in Matlab to analyze the nanocircuit shown in Figure 2. In this section, we present an example of simulation of the nanocircuit shown in Figure 2(a) fed by the Gaussian beam depicted in Figure 3. Figure 4(a) shows the geometry and discretization parameters used in this simulation and Figure 4(b) presents the result of the current distribution along the circuit. The near-field distribution for this example is given in Figure 5.

We observe in these results the stationary behavior in the OTL, which is due to the mismatching in the impedances of nanodipole 2 and OTL. To make a quantitative measure of the impedance matching degree, we calculate approximately the voltage stationary wave ratio (VSWR) near nanodipole 2 as VSWR $=I_{\max } / I_{\min }$, where $I_{\max }$ and $I_{\min }$ are, respectively, the maximum and minimum current magnitude nearest to dipole 2. With this parameter, we calculate the voltage reflection coefficient as $\left|\Gamma_{V}\right|=(\mathrm{VSWR}-1) /(\mathrm{VSWR}+1)$. In this numerical example we obtained $\left|\Gamma_{V}\right|=0.4$.

The next sections present two different analyses of the nanocircuit. In the first analysis, we fix the dimensions of the receiving dipole and focus on the impedance matching problem between the optical transmission line and the emitting dipole; that is, we are interested in the search for the parameters of the nanocircuit which provide maximum energy transferred to the emitting dipole and minimum reflected energy. In the second analysis, we fix the dimensions of the OTL and focus on the coupling problem between the Gaussian beam and the receiving antenna; that is, we are interested in the search for the better dimensions of the receiving dipole which provide maximum receiving energy.

\section{Impedance Matching Analysis}

This section presents a parametric analysis of the impedance matching of the nanocircuit for different values of $h_{2}, a_{h 2}$, $a_{L}$, and $D$. In this analysis, we fixed the dimensions of the receiving dipole $h_{1}=173 \mathrm{~nm}, a_{h 1}=10 \mathrm{~nm}$ and the length of the OTL $L=1200 \mathrm{~nm}$. Figure 6 presents the voltage reflection coefficient $\left|\Gamma_{V}\right|$ versus the total length of the emitting dipole $H_{2}=2 h_{2}+d$ for different values of $D=10,15$, and $20 \mathrm{~nm}$ for the four cases $\left(a_{L}=10 \mathrm{~nm}, a_{h 2}=10 \mathrm{~nm}\right),\left(a_{L}=10 \mathrm{~nm}\right.$, $\left.a_{h 2}=15 \mathrm{~nm}\right),\left(a_{L}=15 \mathrm{~nm}, a_{h 2}=15 \mathrm{~nm}\right)$, and $\left(a_{L}=15 \mathrm{~nm}\right.$, $\left.a_{h 2}=20 \mathrm{~nm}\right)$.

Analyzing these curves we come to some conclusions. We note that the nanocircuits possess in general smaller degree of input impedance matching (higher $\left|\Gamma_{V}\right|$ ) when the gap of the OTL $D$ is increased. The exceptions are the cases of Figures 6(a) and 6(b) in the range $H_{2}<400 \mathrm{~nm}$, where we have a better matching for higher $D$.

We also observe that in general the impedance matching is better when $a_{h 2}>a_{L}$. This means that the values of $\left|\Gamma_{V}\right|$ in Figure 6(d) are smaller than those of Figure 6(c), and the values of $\left|\Gamma_{V}\right|$ in Figure 6(b) are smaller than those of Figure 6(a). This later comparison is only true in the range of $\mathrm{H}_{2}<400 \mathrm{~nm}$.

All these results show that we have many situations of good matching for different values of $D, a_{L}, a_{h 2}$, and $H_{2}$. $\operatorname{Min}\left(\left|\Gamma_{V}\right|\right)$ occurs for larger $H_{2}$, for example, in the cases $D=$ $10 \mathrm{~nm}$ and $H_{2}=640 \mathrm{~nm}$ in Figure 6(a), where $\left|\Gamma_{V}\right| \approx 0.26$, and $D=10 \mathrm{~nm}$ and $H_{2}=610 \mathrm{~nm}$ in Figure $6(\mathrm{~b})$, where $\left|\Gamma_{V}\right| \approx$ 0.35 . Other good results are for smaller $H_{2}$, for example, the case $D=20 \mathrm{~nm}$ and $H_{2}=300 \mathrm{~nm}$ in Figure 6(b), where $\left|\Gamma_{V}\right| \approx 0.31$.

One way to choose the best geometric parameters of the circuit is to consider the case with better impedance matching and efficiency simultaneously. The efficiency of the circuit 


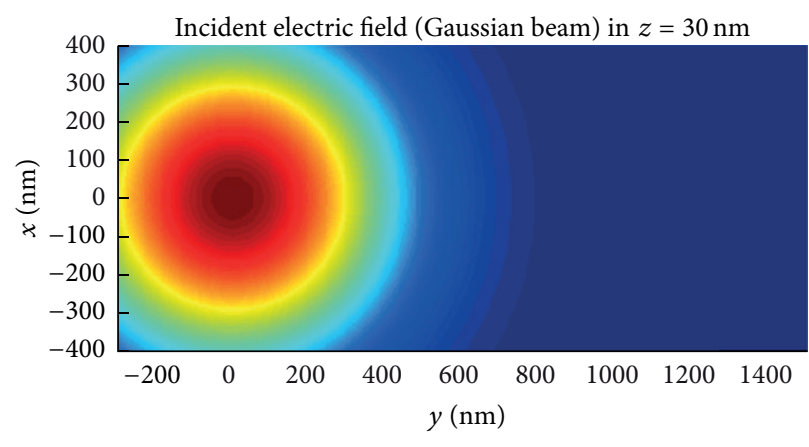

(a)

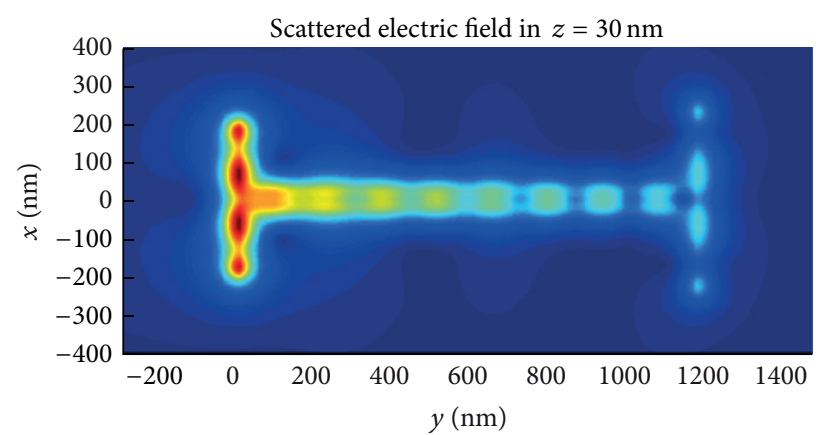

(b)

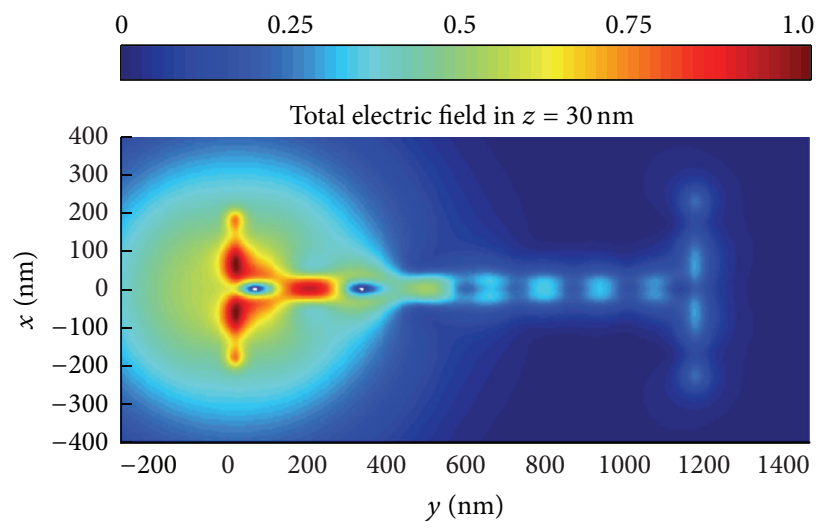

(c)

Figure 5: Normalized distribution of the electric field amplitude at plane $z=30 \mathrm{~nm}$ : (a) incident field of the Gaussian beam abs $\left(E_{x}^{i}\right)$, (b) scattered field $\operatorname{abs}\left(E_{x}^{s}\right)$, and (c) total field $\operatorname{abs}\left(E_{x}\right)$.

depends mainly on the attenuation of the current along the OTL, that is, depends on the loss constant $\alpha$ of the OTL. This parameter is constant for the principal mode that propagates on the OTL and can be obtained approximately by the average inclination of the current versus distance along the OTL. In equation form we have $\alpha=\Delta I / \Delta L$, where $\Delta I$ is the variation of the average amplitude of the current in decibels $(\mathrm{dB})$ along a given distance $\Delta L(\mathrm{~nm})$ in the OTL. With this definition the unit of this parameter is $\mathrm{dB} / \mathrm{nm}$. In the numerical example presented in Figure $4(\mathrm{~b})$ we have $\alpha \approx 0.007393 \mathrm{~dB} / \mathrm{nm}$. This result is very close to that of $\alpha=0.007296 \mathrm{~dB} / \mathrm{nm}$ obtained in [26] where the OTL is similar to our case shown in Figure 4(a) $\left(a_{L}=15 \mathrm{~nm}\right.$ and $\left.D=10 \mathrm{~nm}\right)$.

To better understand the behavior of the impedance matching and efficiency characteristic of the results presented in Figure 6, we plot in Figures 7 and 8 the current distributions for different geometric parameters. Figure 7 shows the currents for two cases with the same $a_{L}=10 \mathrm{~nm}$ but different voltage reflection coefficient of $\left|\Gamma_{V}\right|=0.31$ and 0.67 . In these results we observe a higher stationary wave for the case $\left|\Gamma_{V}\right|=0.67$ than for the case $\left|\Gamma_{V}\right|=0.31$. This shows that our approximate method to calculate $\left|\Gamma_{V}\right|$ provides a good measure of the degree of impedance matching. We also observe that the two cases present the same attenuation along the circuit (i.e., in both cases one has approximately the same loss constant $\alpha=0.0111 \mathrm{~dB} / \mathrm{nm}$ ) because the OTL are constructed with the same radius of wires $a_{L}=10 \mathrm{~nm}$.

Figure 8 presents the current distribution for two cases with good impedance matching $\left|\Gamma_{V}\right|=0.26$ and 0.36 but with different loss constant of $\alpha=0.0119 \mathrm{~dB} / \mathrm{nm}$ and $0.0084 \mathrm{~dB} / \mathrm{nm}$, respectively. This difference is mainly due to the difference of the radius $a_{L}$ of the OTL. For lower values of $a_{L}$ the attenuation is higher in OTL and this result is similar to that observed in RF-microwave regimes. This can be explained by the surface impedance model of (2), where smaller radius produces higher $Z_{s}$ and, consequently, higher loss in the conductors.

\section{Excitation Analysis}

4.1. Analysis of Receiving Dipole. In this section, we investigate the response of the isolated receiving dipole illuminated by a Gaussian beam polarized along nanodipole's axis (left side of Figure 3). We search the values of $H_{1}$ and $a_{h 1}$ which maximize the coupling between the beam and the antenna, that is, maximize the resonant response of the dipole. Figure 9 shows the variation of the current amplitude in the middle of the nanodipole $\operatorname{abs}\left(I_{c}\right)$ in function of the total length $H_{1}$ and radius $a_{h 1}$. For comparison, this figure shows also the results calculated with the software Comsol [30]. These 


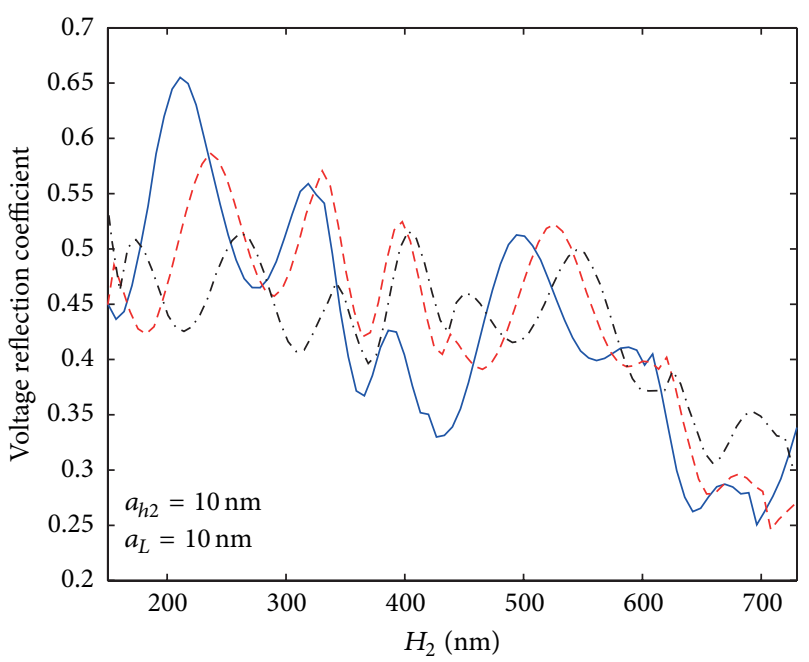

(a)

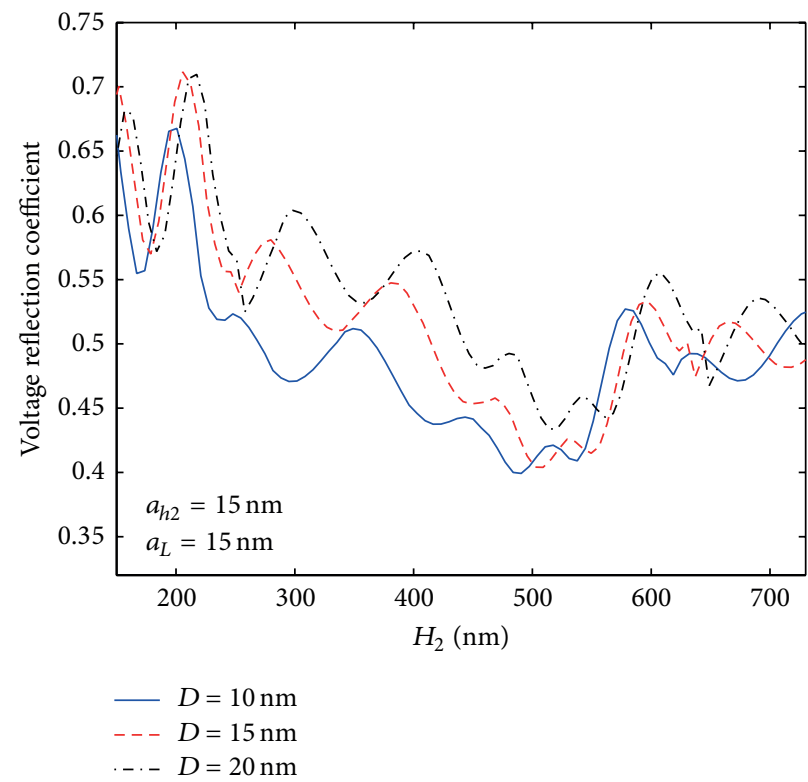

(c)

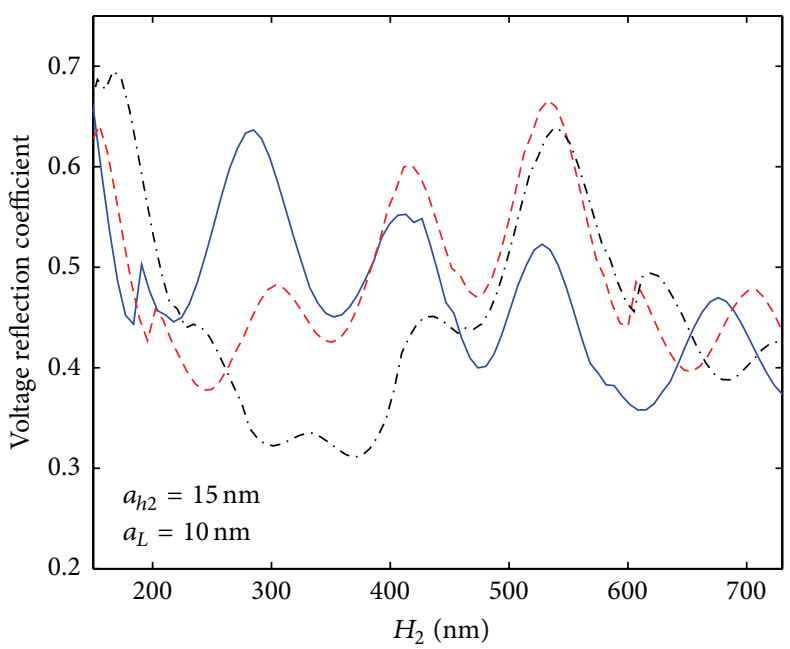

(b)

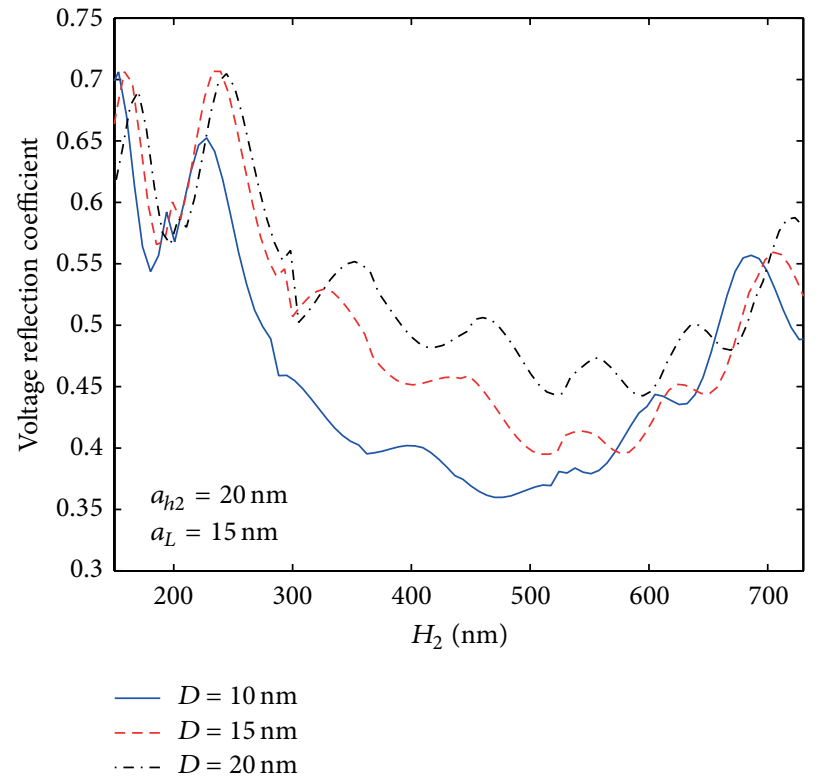

(d)

Figure 6: Voltage reflection versus $H_{2}=2 h_{2}+d$ for different values of $D=10,15$, and $20 \mathrm{~nm}$. (a) $a_{L}=10 \mathrm{~nm}$ and $a_{h 2}=10 \mathrm{~nm}$. (b) $a_{L}=10 \mathrm{~nm}$ and $a_{h 2}=15 \mathrm{~nm}$. (c) $a_{L}=15 \mathrm{~nm}$ and $a_{h 2}=15 \mathrm{~nm}$. (d) $a_{L}=15 \mathrm{~nm}$ and $a_{h 2}=20 \mathrm{~nm}$.

currents are approximately the same input currents when a given nanodipole is connected to the input terminal of the nanocircuit in Figure 1. Thus when this current has the maximum value the power entering the OTL is also maximal.

The results of Figure 9 show that the maximum induced current $I_{c}$ occurs for the resonant lengths $H_{1}$ corresponding to the resonances $\lambda / 2$ and $3 \lambda / 2$. The values of these resonant lengths are given in Table 1. One example of the characteristic field distributions in these resonances is presented in Figure 10 where $H_{1}=111.2 \mathrm{~nm}$ and $a_{h 1}=10 \mathrm{~nm}$. Also, the results of Figure 9 show that for higher values of $a_{h 1}$ we have larger $I_{c}$ and the resonant lengths $H_{1}$ are increased. We observed that the resonance $\lambda / 2$ produces higher current in the nanodipole than in the resonance $3 \lambda / 2$. We can conclude that we have a better coupling when the nanodipole operates in the first resonance and possesses a large radius. The latter condition implies high effective area of the nanodipole [31].

4.2. Voltage Reflection Coefficient. In this section we analyze the voltage reflection coefficient of the nanocircuit in Figure 1 where we use the resonant receiving nanodipoles with dimensions given in Table 1. Figure 11 presents the variation of $\left|\Gamma_{V}\right|$ in function of $H_{2}$ for the first resonance $\lambda / 2$ and the second resonance $3 \lambda / 2$, with different values of $a_{h 1}$. In these simulations we fixed the following parameters of the OTL and 
TABLE 1: Resonant length $H_{1}$ of the receiving dipole.

\begin{tabular}{lccc}
\hline & $a_{h 1}=10 \mathrm{~nm}$ & $a_{h 1}=15 \mathrm{~nm}$ & $a_{h 1}=20 \mathrm{~nm}$ \\
\hline$H_{1}$ for $\lambda / 2$ resonance $(\mathrm{nm})$ & 111.2 & 148.5 & 176.6 \\
$H_{1}$ for $3 \lambda / 2$ resonance $(\mathrm{nm})$ & 385.3 & 528.6 & 637.7 \\
\hline
\end{tabular}

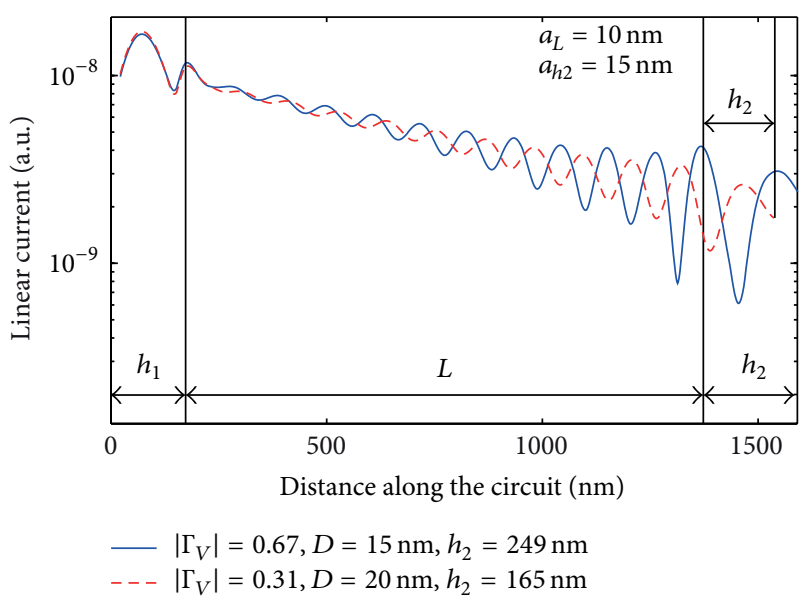

FIGURE 7: Current distributions of two circuits with the same values of $a_{L}=10 \mathrm{~nm}$ possessing different voltage reflection coefficient $\left(\left|\Gamma_{V}\right|=0.31\right.$ and 0.67$)$.

emitting dipole: $a_{L}=15 \mathrm{~nm}, a_{h 2}=20 \mathrm{~nm}, L=1200 \mathrm{~nm}$, and $N_{L}=40$. We choose these parameters because they presented a better impedance matching and lower attenuation.

The results of Figure 11 show that the reflection coefficient does not vary significantly with the radius $a_{h 1}$ and length $H_{1}$ for the first resonance (Figure 11(a)). However, for the second resonance (Figure 11(b)) we have a better input impedance matching for larger values of $H_{1}$ and $a_{h 1}$. The best impedance matching is obtained for the case $a_{h 1}=20 \mathrm{~nm}$ and $H_{1}=637.7 \mathrm{~nm}$, where we have $\left|\Gamma_{V}\right|=0.21$. We also note that the results of impedance matching for the second resonance (Figure 11(b)) are better than those of the first resonance (Figure 11(a)). These results prove that the degree of impedance matching along the circuit not only is a function of the OTL and the emitting dipole but also depends on the geometry of the receiving dipole.

4.3. Current and Field Distribution. Figure 12 shows the current distribution along the circuit for the best cases of $\left|\Gamma_{V}\right|$ presented in Figure 11. In Figure 12(a) we plot the current for $H_{2}=470 \mathrm{~nm}$ which corresponds approximately to the minimum voltage reflection coefficient of the curves in Figure 11(a). In Figure 12(b) we plot the current for $\mathrm{H}_{2}=$ $500 \mathrm{~nm}$, which corresponds to the minimum voltage reflection coefficient of the curves of Figure 11(b).

We observe that the amplitude of the input current in the nanocircuit is in general in accordance with the results presented in Figure 9 for the isolated receiving dipole, where for larger $H_{1}$ and $a_{h 1}$ we have higher current along the circuit for both resonances. However, for fixed $a_{h 1}=10 \mathrm{~nm}$ the first

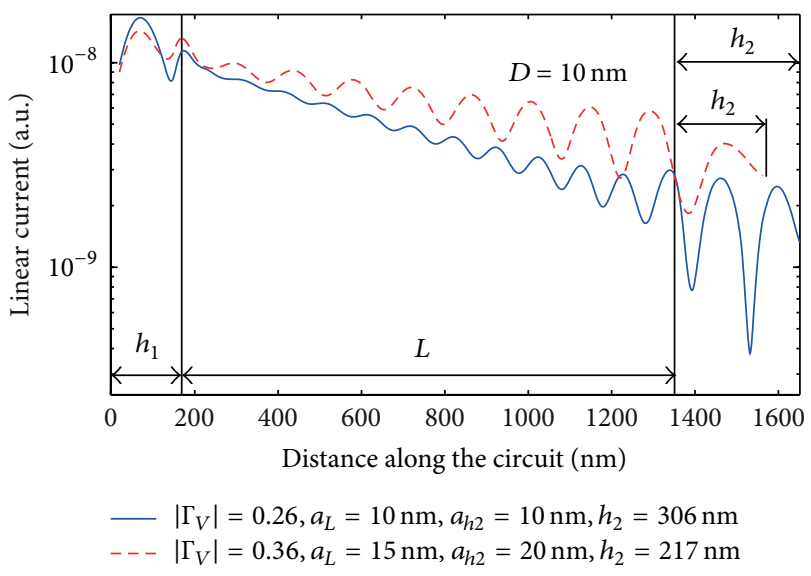

FIGURE 8: Current distributions of two nanocircuits with good voltage reflection coefficient $\left(\left|\Gamma_{V}\right|=0.26\right.$ and 0.36$)$ possessing different values of $a_{L}$.

resonance (Figure 12(a)) has the smaller input current amplitude as compared to the second resonance (Figure 12(b)). For $a_{h 1}=15 \mathrm{~nm}$, the first resonance (Figure 12(a)) presents the same level of the input current amplitude as that of the second resonance (Figure 12(b)). And for $a_{h 1}=20 \mathrm{~nm}$ the first resonance (Figure 12(a)) presents higher input current amplitude than that of the second resonance (Figure 12(b)). However, the cases of Figure 12(b) give a better impedance matching (lower values of $\left|\Gamma_{V}\right|$ ) than the cases of Figure 12(a).

We conclude with this analysis that a good impedance matching does not necessarily mean a good efficiency in the receiving dipole, that is, higher input current amplitude. For example, the case of $a_{h 1}=20 \mathrm{~nm}$ and $H_{1}=176.6 \mathrm{~nm}$ (Figure 12(a)) presents a higher intensity of the input current but a smaller voltage reflection coefficient than the case of $a_{h 1}=20 \mathrm{~nm}$ and $H_{1}=637.7 \mathrm{~nm}$ (Figure 12(b)). The total electric field distribution at plane $z=30 \mathrm{~nm}$ of these two opposite situations is shown in Figure 13. We can see in this figure the differences in the field intensity, showing higher values in the emitting dipole for case of the first resonance (Figure 13(a)).

\section{Conclusions}

In this paper, we presented a theoretical analysis of the impedance matching and excitation characteristics of a plasmonic optical nanocircuit fed by a Gaussian beam, which is composed of a receiving and an emitting dipole connected by a two-wire optical transmission line. The method of moments with equivalent surface impedance of the conductors was used for numerical calculations.

In the impedance matching analysis, we presented a parametric study of the reference circuit for different geometrical dimensions such as line and emitting dipole radius, gap distance of the line, and length of emitting dipole. We observed that better impedance matching is obtained when the radius of the emitting dipole is larger than the radius of the OTL. We also noted that the impedance matching can 


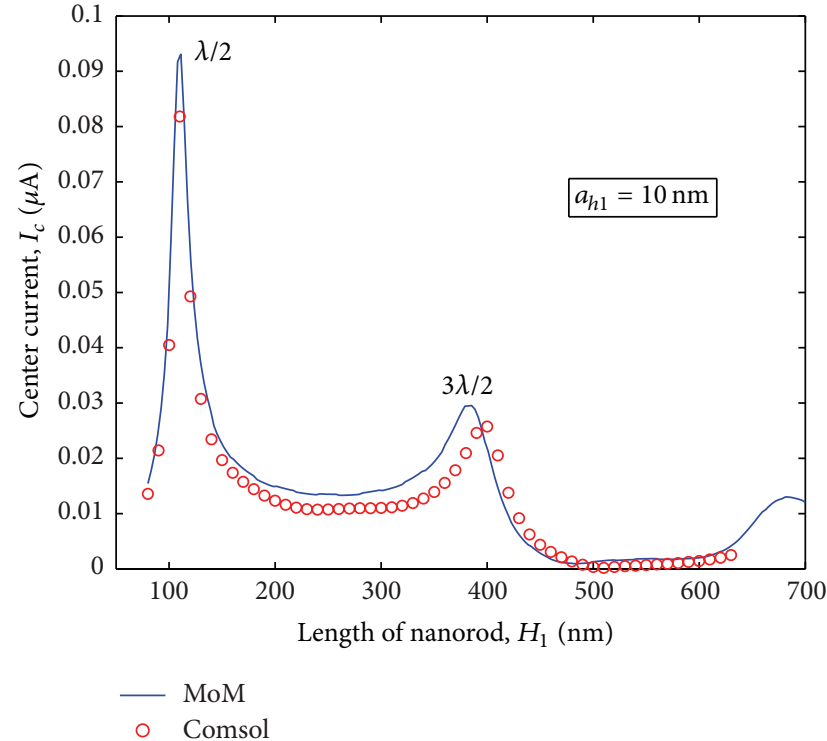

(a)

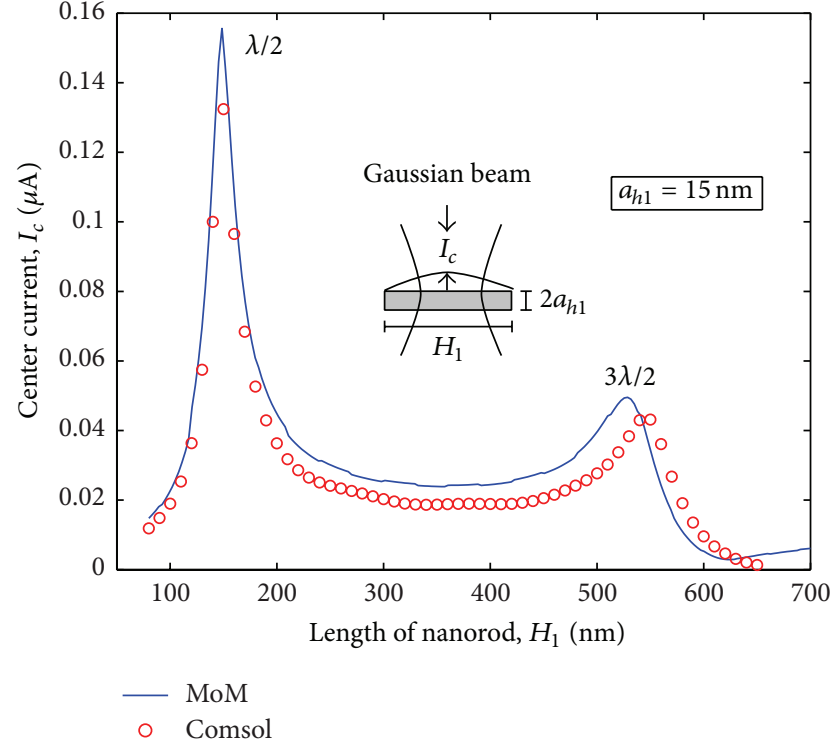

(b)

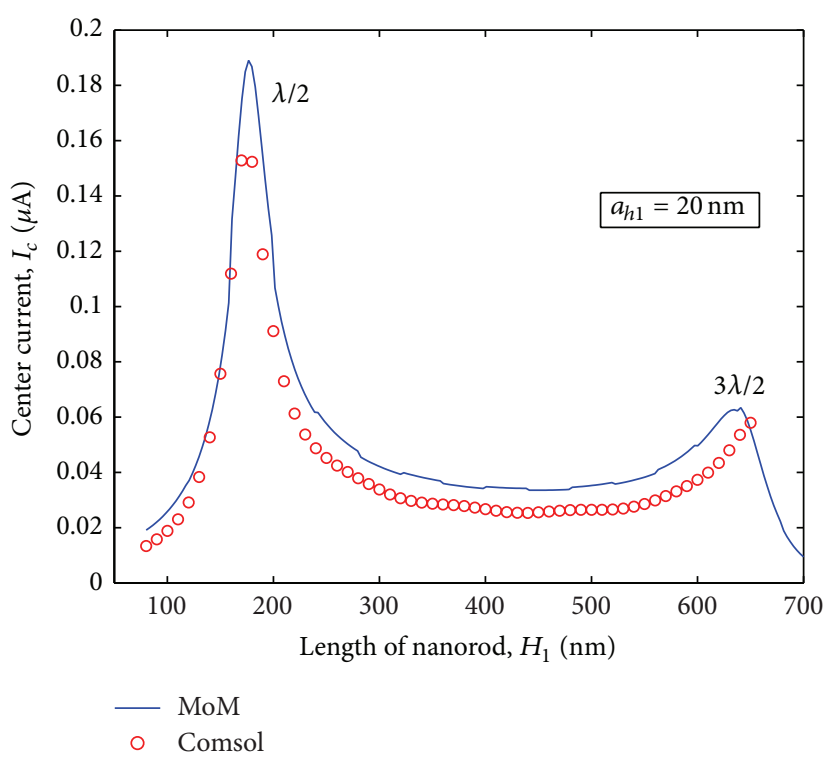

(c)

FigURE 9: Variation of current amplitude in middle of nanorod $\left(I_{c}\right)$ in function of total length $H_{1}$ and radius $a_{h 1}$.

be optimized varying the gap distance of the line and length of emitting dipole, and there are many possible solutions for good impedance matching. With respect to the attenuation losses in the line, we observed that a good impedance matching does not necessarily mean a good transmission efficiency along the OTL. In other words, we can have circuits with the same degree of impedance matching but with different transmission efficiency, that is, with different attenuation losses. This occurs because the efficiency is mainly a function of the radius of the OTL, where for a smaller radius the conductors possess a larger equivalent surface impedance and consequently higher conduction loss.
In the excitation analysis, our results show that receiving dipoles with larger radius produce a better impedance matching and a higher input current in the circuit. However, good reception efficiency of the receiving dipole does not necessarily mean good impedance matching in the nanocircuit. In general, receiving dipole in the first resonance presents better reception efficiency but poor impedance matching in comparison with that of the second resonance. This means that to design an efficient circuit one has to make a compromise between reception efficiency, transmission efficiency, and impedance matching. However, in terms of efficiency, the best circuit that we have obtained is that presented in 


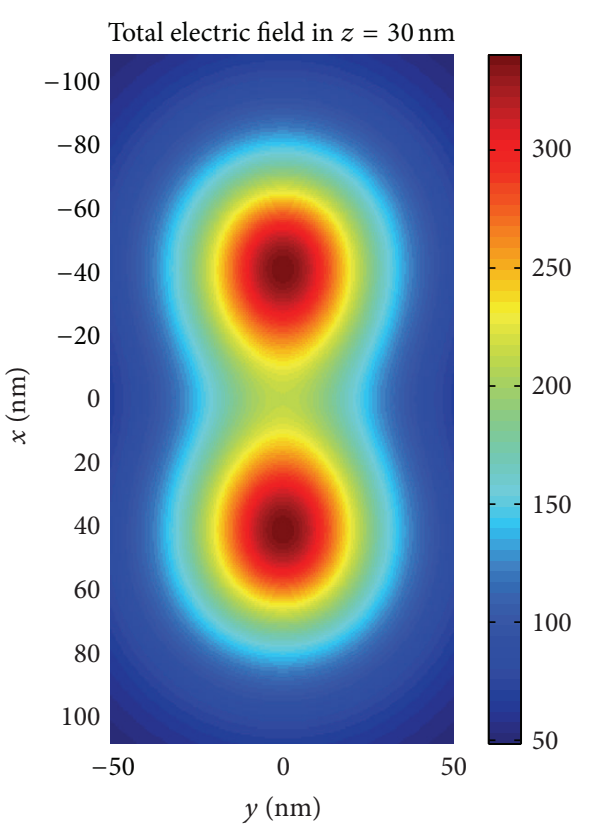

(a)

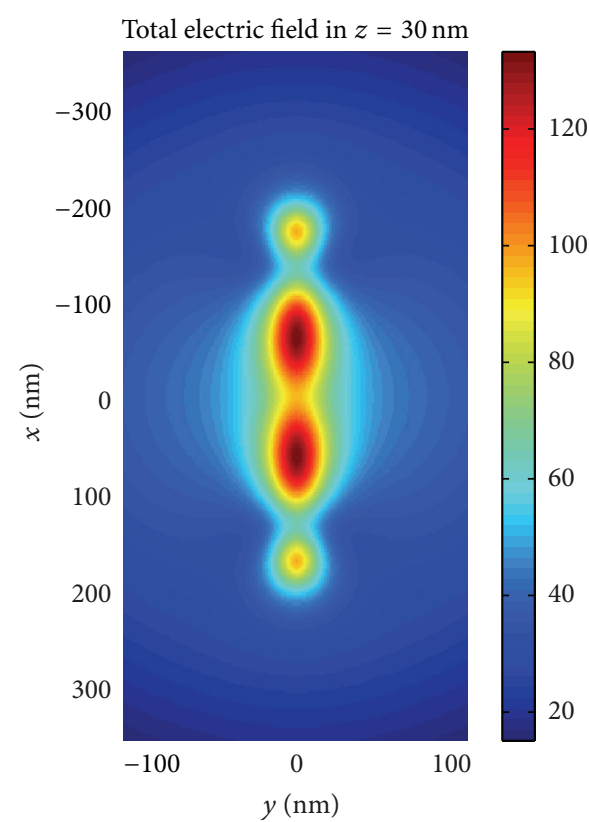

(b)

FIGURE 10: Electric field distribution $(\mathrm{V} / \mathrm{m})$ at plane $z=30 \mathrm{~nm}$ of the nanodipoles with $a_{h 1}=10 \mathrm{~nm}$. (a) $\lambda / 2$ resonance $\left(H_{1}=111.2 \mathrm{~nm}\right)$. (b) $3 \lambda / 2$ resonance $\left(H_{1}=385.3 \mathrm{~nm}\right)$.

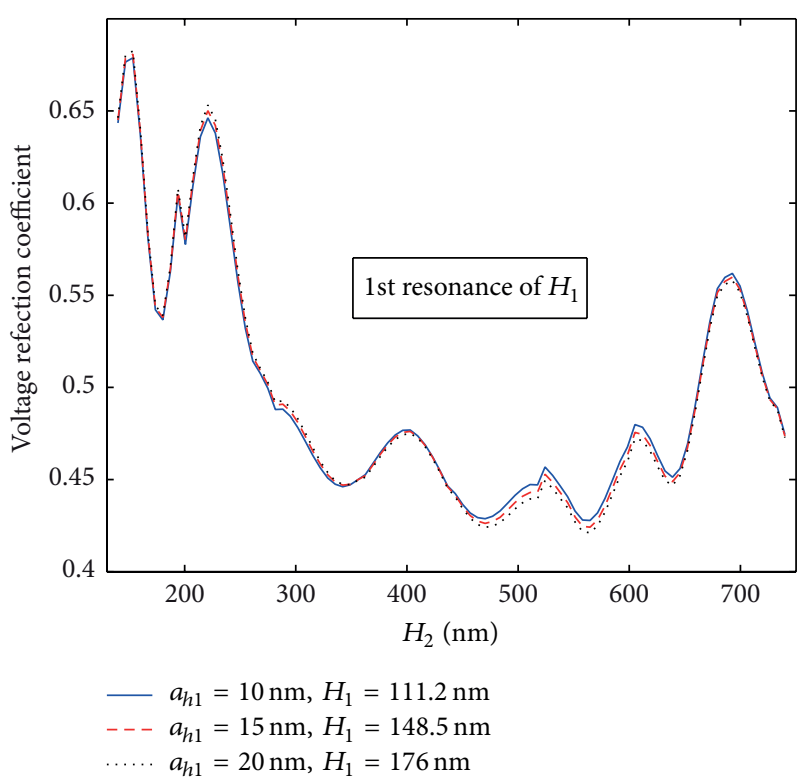

(a)

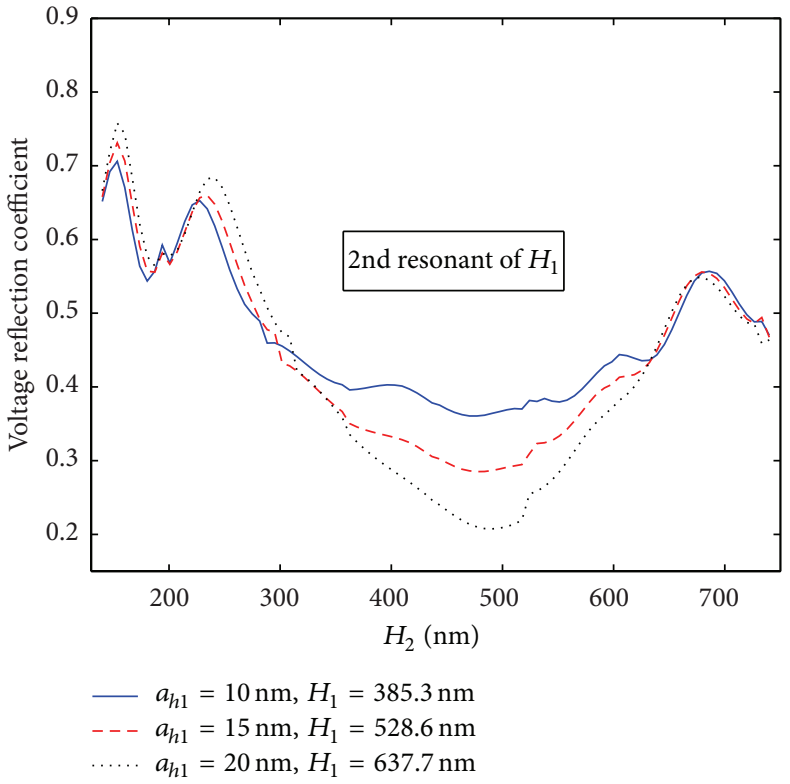

(b)

FIGURE 11: Voltage reflection coefficient of nanocircuit versus $H_{2}$ for different radius $a_{h 1}$ of receiving nanodipole in different resonances. (a) First resonance $\lambda / 2$. (b) Second resonance $3 \lambda / 2$.

Figure 13(a), where the level of the signal that arrives in the emitting dipole is the maximum one obtained. This situation is only accomplished when the receiving dipole operates in the first resonance and the emitting dipole operates in the second resonance.
The analysis presented here can be useful as guidelines to design efficient plasmonic optical nanocircuits for applications in integrated nanophotonics for optical signal data processing. In future works, we will investigate the overall efficiency of the nanocircuit and consider some other 


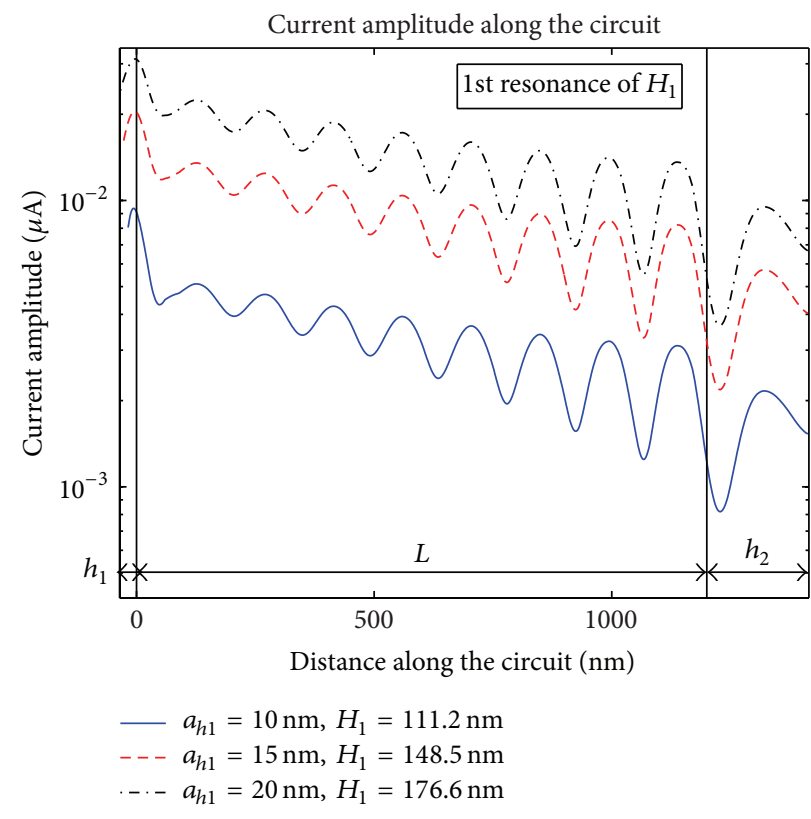

(a)

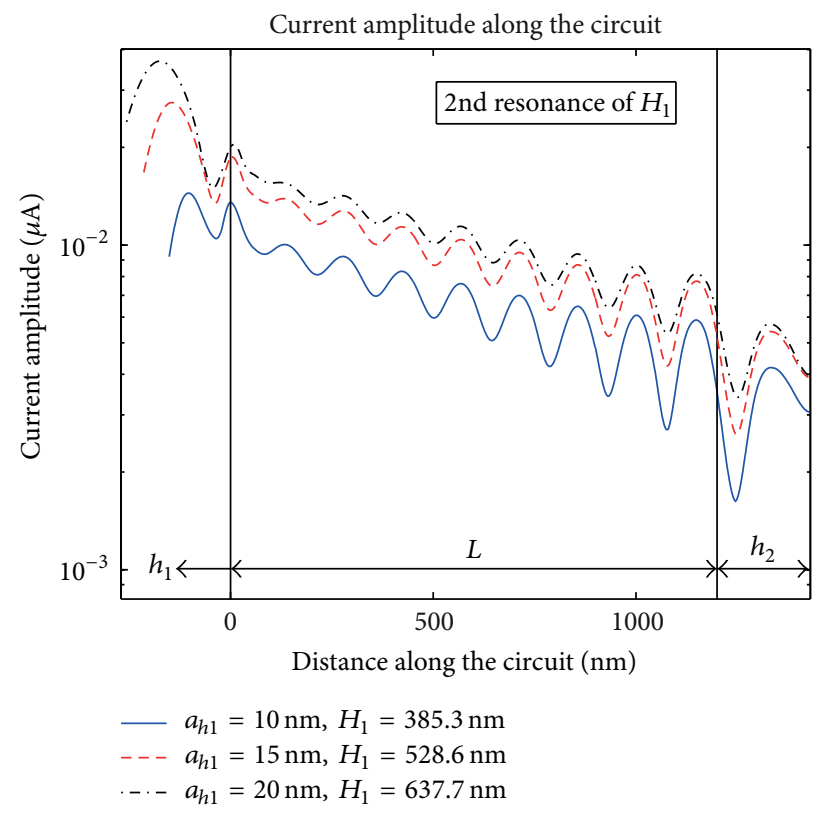

(b)

FIGURE 12: Current distribution along nanocircuit for different receiving dipole in different resonances. (a) Results for the first resonance $(\lambda / 2)$, where the parameters used in these curves are as follows: $a_{L}=15 \mathrm{~nm}, a_{h 2}=20 \mathrm{~nm}$, and $H_{2}=470 \mathrm{~nm}$. (b) Results for the second resonance $(3 \lambda / 2)$, where the parameters used in these curves are as follows: $a_{L}=15 \mathrm{~nm}, a_{h 2}=20 \mathrm{~nm}$, and $H_{2}=500 \mathrm{~nm}$.

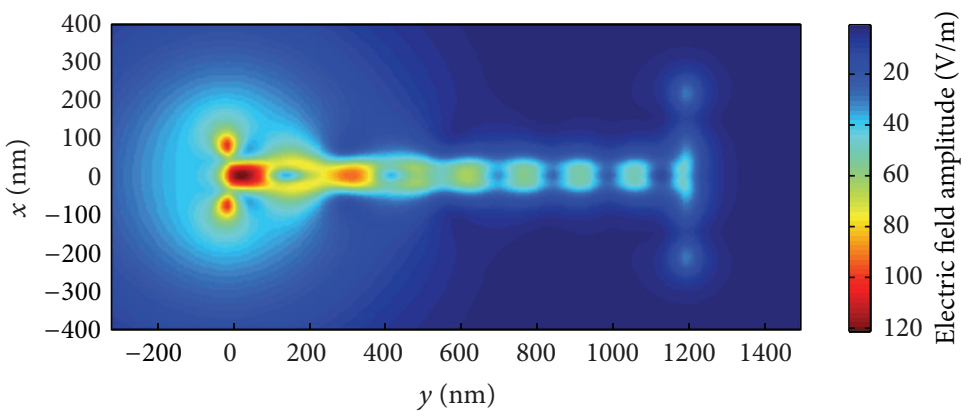

(a)

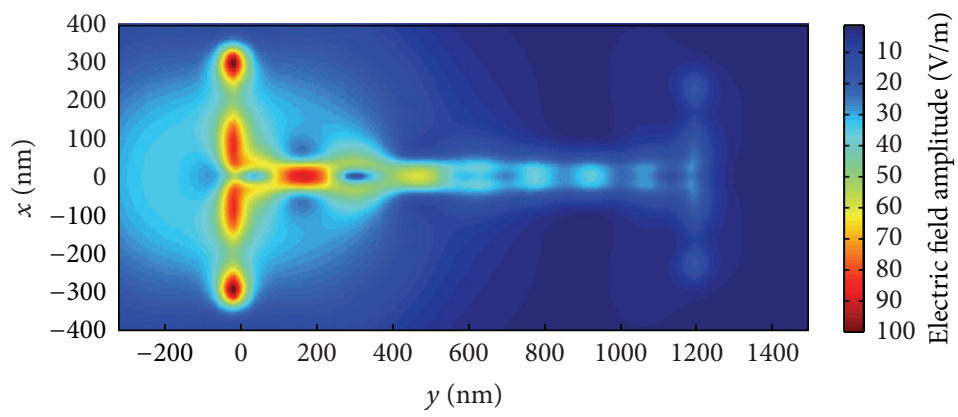

(b)

FIGURE 13: Total (scattered and incident) near electric field distribution at plane $z=30 \mathrm{~nm}$ of nanocircuit for the best cases presented in Figure 12. (a) Receiving dipole at first resonance with $H_{1}=176.6 \mathrm{~nm}$ and $a_{h 1}=20 \mathrm{~nm}$. The voltage reflection coefficient in circuit in this case is $\left|\Gamma_{V}\right|=0.42$. (b) Receiving dipole at second resonance with $H_{1}=637.7 \mathrm{~nm}$ and $a_{h 1}=20 \mathrm{~nm}$. The voltage reflection coefficient in circuit in this case is $\left|\Gamma_{V}\right|=0.21$. 
geometries of nanoantennas to optimize the energy transfer in the circuit.

\section{Conflict of Interests}

The authors declare that there is no conflict of interests regarding the publication of this paper.

\section{Acknowledgments}

This work was supported by the Brazilian agencies PROPESP/ UFPA, FADESP, and FAPESPA.

\section{References}

[1] L. Novotny and B. Hecht, Principles of Nano-Optics, Cambridge, New York, NY, USA, 2006.

[2] A. Alù and N. Engheta, "Theory, modeling and features of optical nanoantennas," IEEE Transactions on Antennas and Propagation, vol. 61, no. 4, pp. 1508-1517, 2013.

[3] P. Biagioni, J.-S. Huang, and B. Hecht, "Nanoantennas for visible and infrared radiation," Reports on Progress in Physics, vol. 75, no. 2, Article ID 024402, 2012.

[4] L. Novotny and N. van Hulst, "Antennas for light," Nature Photonics, vol. 5, no. 2, pp. 83-90, 2011.

[5] S. A. Maier, Plasmonics: Fundamentals and Applications, Springer, 2007.

[6] M. L. Brongersma and P. G. Kik, Surface Plasmon Nanophotonics, Springer, 2007.

[7] R. D. Grober, R. J. Schoelkopf, and D. E. Prober, "Optical antenna: towards a unity efficiency near-field optical probe," Applied Physics Letters, vol. 70, no. 11, pp. 1354-1356, 1997.

[8] D. W. Pohl, "Near field optics seen as an antenna problem," in Proceedings of the 2nd Asia-Pacific Workshop on Near Field Optics, pp. 9-21, Beijing, China, 1999.

[9] T. H. Taminiau, F. B. Segerink, and N. F. van Hulst, "A monopole antenna at optical frequencies: single-molecule near-field measurements," IEEE Transactions on Antennas and Propagation, vol. 55, no. 11, pp. 3010-3017, 2007.

[10] K. Iwami, T. Ono, and M. Esashi, "Optical near-field probe integrated with self-aligned bow-tie antenna and electrostatic actuator for local field enhancement," Journal of Microelectromechanical Systems, vol. 15, no. 5, pp. 1201-1208, 2006.

[11] V. Giannini, J. A. Sánchez-Gil, O. L. Muskens, and J. G. Rivas, "Electrodynamic calculations of spontaneous emission coupled to metal nanostructures of arbitrary shape: nanoantennaenhanced fluorescence," Journal of the Optical Society of America B: Optical Physics, vol. 26, no. 8, pp. 1569-1577, 2009.

[12] I. Kocakarin and K. Yegin, "Surface plasmon-enhanced nanoantenna for localized fluorescence," International Journal of Antennas and Propagation, vol. 2012, Article ID 592842, 7 pages, 2012.

[13] J.-W. Liaw, "Analysis of a bowtie nanoantenna for the enhancement of spontaneous emission," IEEE Journal on Selected Topics in Quantum Electronics, vol. 14, no. 6, pp. 1441-1447, 2008.

[14] K. Q. Costa and V. A. Dmitriev, "Bowtie nanoantennas with polynomial sides in the excitation and emission regimes," Progress In Electromagnetics Research B, no. 32, pp. 57-73, 2011.

[15] H. Wang, C. T. Chong, and L. Shi, "Optical antennas and their potential applications to 10 Terabit/in ${ }^{2}$ recording," in
Proceedings of the Optical Data Storage Topical Meeting (ODS '09), pp. 16-18, May 2009.

[16] D. P. Fromm, A. Sundaramurthy, A. Kinkhabwala, P. J. Schuck, G. S. Kino, and W. E. Moerner, "Exploring the chemical enhancement for surface-enhanced Raman scattering with $\mathrm{Au}$ bowtie nanoantennas," Journal of Chemical Physics, vol. 124, no. 6, Article ID 061101, 2006.

[17] M. F. Garcia-Parajo, "Optical antennas focus in on biology," Nature Photonics, vol. 2, no. 4, pp. 201-203, 2008.

[18] T. H. Taminiau, F. D. Stefani, and N. F. van Hulst, "Enhanced directional excitation and emission of single emitters by a nanooptical Yagi-Uda antenna," Optics Express, vol. 16, no. 14, pp. 10858-10866, 2008.

[19] H. F. Hofmann, T. Kosako, and Y. Kadoya, "Design parameters for a nano-optical Yagi-Uda antenna," New Journal of Physics, vol. 9, article 217, 2007.

[20] E. Cubukcu, N. Yu, E. J. Smythe, L. Diehl, K. B. Crozier, and F. Capasso, "Plasmonic laser antennas and related devices," IEEE Journal on Selected Topics in Quantum Electronics, vol. 14, no. 6, pp. 1448-1461, 2008.

[21] H. A. Atwater and A. Polman, "Plasmonics for improved photovoltaic devices," Nature Materials, vol. 9, no. 3, pp. 205213, 2010.

[22] K. Q. Le, "Broadband light trapping in thin organic photovoltaic cells using plasmonic resonant antennas," Journal of Applied Physics, vol. 114, Article ID 084504, 2013.

[23] D. K. Gramotnev and S. I. Bozhevolnyi, "Plasmonics beyond the diffraction limit," Nature Photonics, vol. 4, no. 2, pp. 83-91, 2010.

[24] H. Pahlevaninezhad, T. E. Darcie, and B. Heshmat, "Two-wire waveguide for terahertz," Optics Express, vol. 18, no. 7, pp. 74157420, 2010.

[25] N. Engheta, "Circuits with light at nanoscales: optical nanocircuits inspired by metamaterials," Science, vol. 317, no. 5845, pp. 1698-1702, 2007.

[26] J.-S. Huang, T. Feichtner, P. Biagioni, and B. Hecht, "Impedance matching and emission properties of nanoantennas in an optical nanocircuit," Nano Letters, vol. 9, no. 5, pp. 1897-1902, 2009.

[27] K. Q. da Costa, V. A. Dmitriev, and G. L. Silvano, "Impedance matching analysis of an optical nanocircuit fed by an aperture probe," Journal of Microwaves, Optoelectronics and Electromagnetic Applications, vol. 12, pp. 42-56, 2013.

[28] K. Zhang and D. Li, Electromagnetic Theory for Microwaves and Optoelectronics, Springer, New York, NY, USA, 2nd edition, 2007.

[29] G. W. Hanson, "On the applicability of the surface impedance integral equation for optical and near infrared copper dipole antennas," IEEE Transactions on Antennas and Propagation, vol. 54, no. 12, pp. 3677-3685, 2006.

[30] COMSOL Multiphysics 4.2a, COMSOL Inc., http://www.comsol.com.

[31] C. A. Balanis, Antenna Theory: Analysis and Design, John Wiley \& Sons, New York, NY, USA, 3nd edition, 2005. 

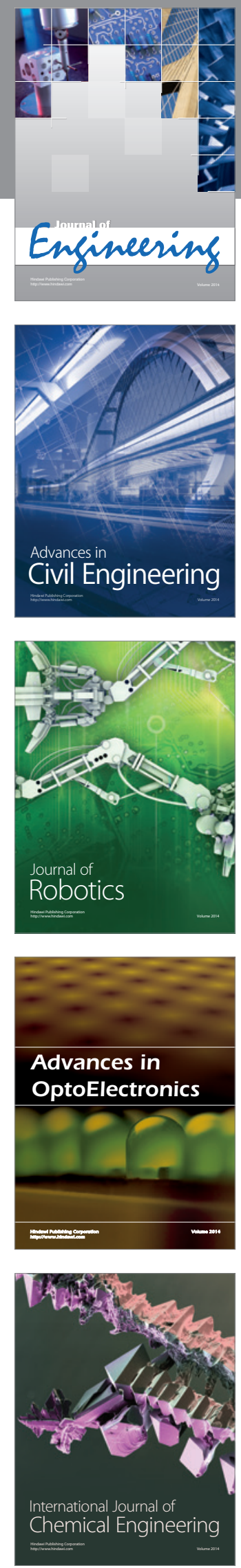

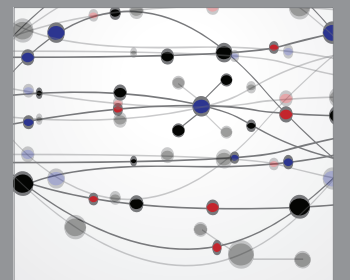

The Scientific World Journal
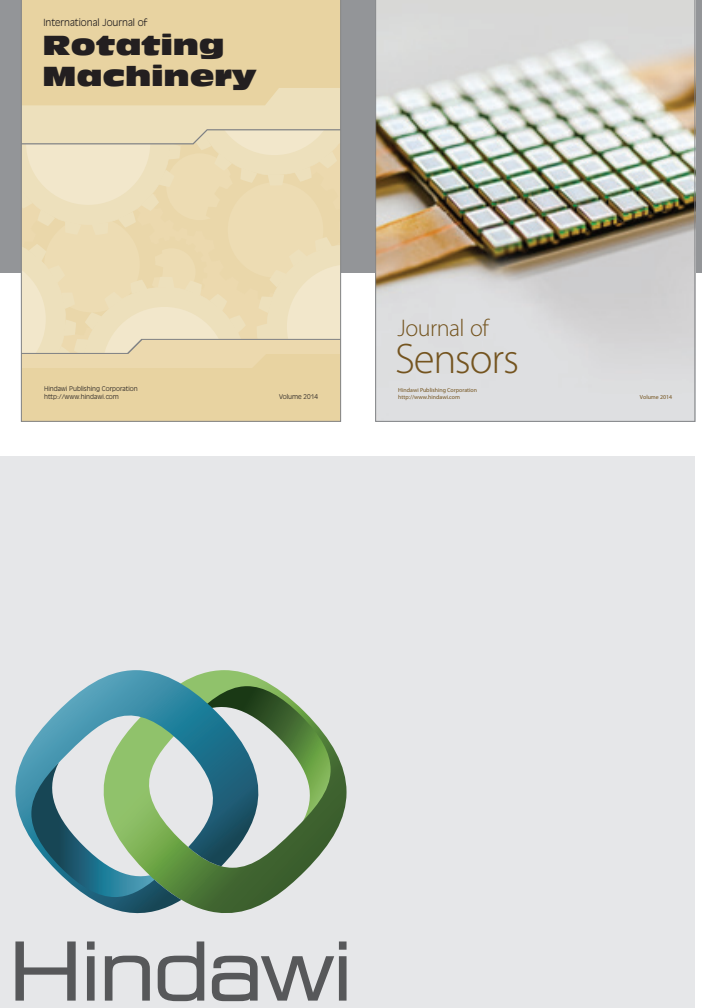

Submit your manuscripts at http://www.hindawi.com
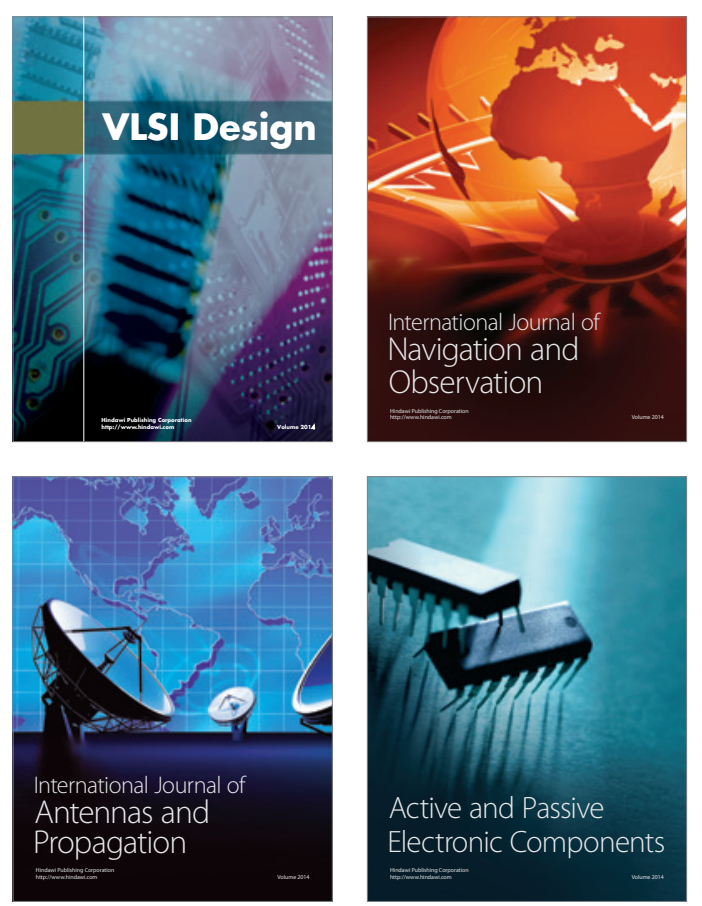
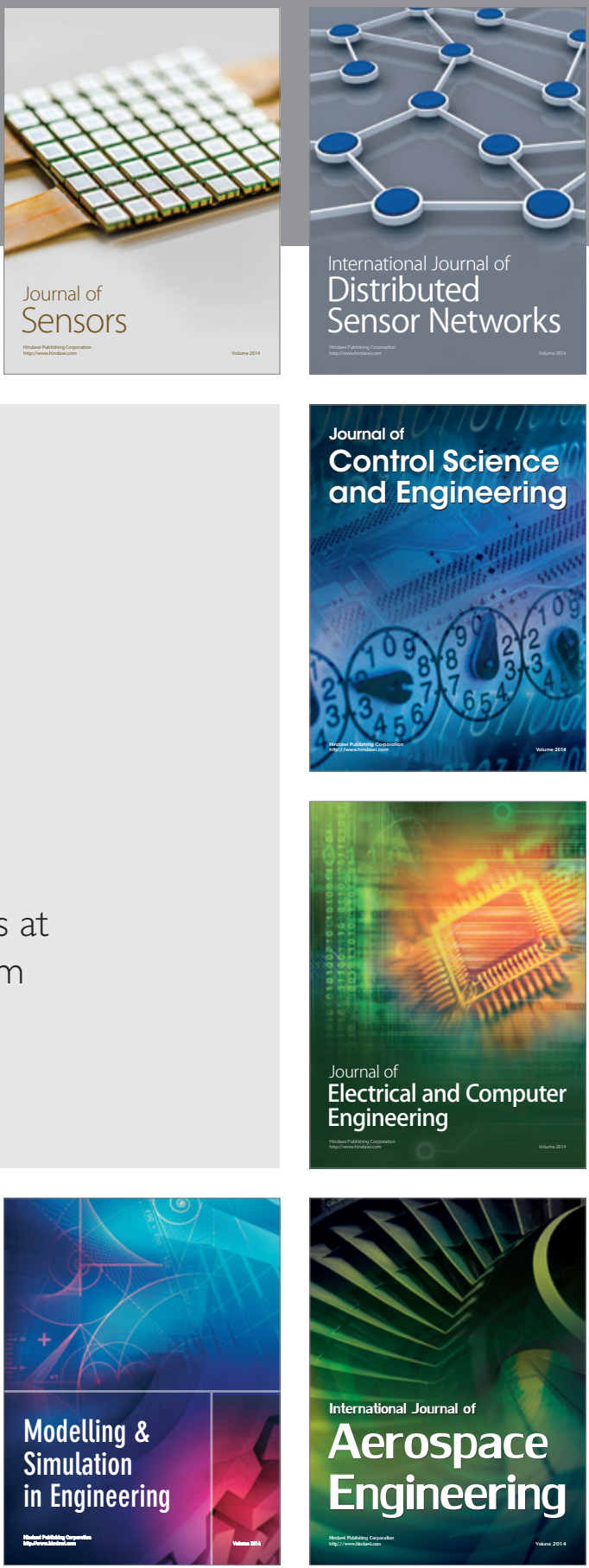

Journal of

Control Science

and Engineering
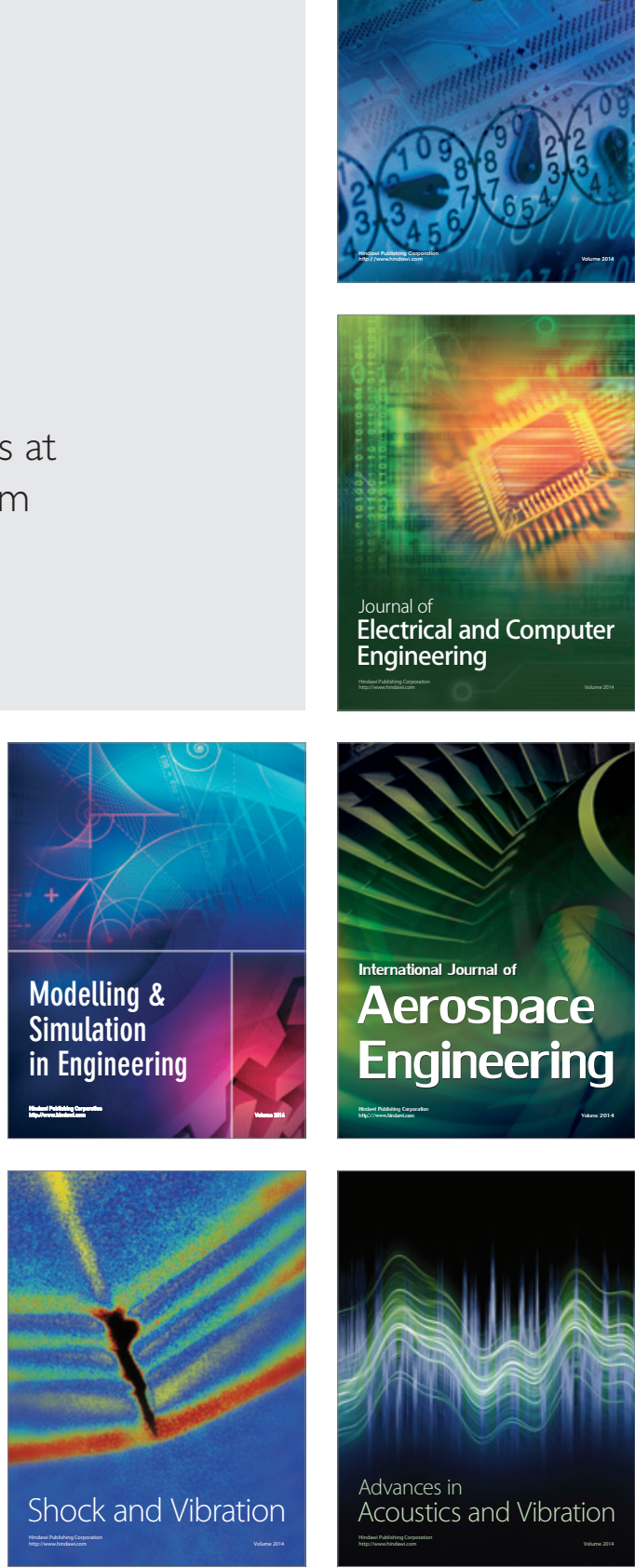